\title{
Impact of Elagolix on Workplace and Household Productivity Among Women with Moderate to Severe Pain Associated with Endometriosis: A Pooled Analysis of Two Phase III Trials
}

\author{
Eric S. Surrey ${ }^{1} \cdot$ Ahmed M. Soliman $^{2} \cdot$ Hannah L. Palac $^{2} \cdot$ Sanjay K. Agarwal ${ }^{3}$
}

Published online: 25 October 2019

(c) The Author(s) 2019

\begin{abstract}
Background Endometriosis profoundly impairs women's workplace and household productivity.

Objective The aim of this study was to evaluate the impact of elagolix on endometriosis-related workplace and household productivity losses.

Methods Data were pooled from two phase III trials of women aged 18-49 years with moderate to severe endometriosis-associated pain treated for 6 months with elagolix $150 \mathrm{mg}$ daily (QD), $200 \mathrm{mg}$ twice daily (BID), or placebo. The Health-Related Productivity Questionnaire was administered at baseline, Month 3, and Month 6 to determine workplace and household absenteeism and presenteeism. Productivity changes from baseline were compared between placebo and elagolix doses via analysis of covariance. Results Workplace analyses included 1270 employed women and household analyses included 1565 women. At baseline, women reported average weekly losses of 16 workplace hours, 8.3 household work hours, $45 \%$ of scheduled work, and $64 \%$ of planned household chores. At Month 6, treatment with elagolix $150 \mathrm{mg}$ QD or $200 \mathrm{mg}$ BID increased productive workplace hours by 1.7 (95\% CI 0.1-3.4; $p=0.041)$ and $5.4 \mathrm{~h}(95 \% \mathrm{CI} 3.7-7.1 ; p<0.001)$ relative to placebo, corresponding to gains of 5.2\% (95\% CI $0.7-9.7 ; p=0.022)$ and $14.6 \%$ (95\% CI 10.0-19.1; $p<0.001)$ of scheduled work, respectively. Both elagolix doses improved household productivity at Month 6 by 1.7 (95\% CI 0.7-2.7) and 3.1 (95\% CI 2.1-4.0) hours relative to placebo (both $p<0.001$ ), with increases of 8.8\% (95\% CI 3.5-14.1; $p=0.001$ ) and 20.4\% (95\% CI 15.1-25.6; $p<0.001$ ) of planned household work.

Conclusions Treatment with elagolix improved endometriosis-related workplace and household productivity impairments. Trial Registration ELARIS EM-I (NCT01620528) and ELARIS EM-II (NCT01931670)
\end{abstract}

Data presented in this communication were reported in part at the International Society for Pharmacoeconomics and Outcomes Research Annual Conference, May 18-22, 2019, New Orleans, LA, USA.

\section{Eric S. Surrey}

ESurrey@colocrm.com

1 Colorado Center for Reproductive Medicine, 10290 RidgeGate Circle, Lone Tree, CO 80124, USA

2 AbbVie Inc., North Chicago, IL, USA

3 Center for Endometriosis Research and Treatment, University of California, San Diego, CA, USA

\section{Key Points for Decision Makers}

Endometriosis and its associated pain symptoms have been shown to profoundly reduce women's health-related quality of life as well as impair employment-based and household productivity.

This is the first pooled analysis of data from randomized, placebo-controlled studies investigating the impact of treatment on workplace and household productivity impairment in a large cohort of women with moderate to severe endometriosis-associated pain.

Women with moderate to severe endometriosis-associated pain treated with elagolix $150 \mathrm{mg}$ daily or $200 \mathrm{mg}$ twice daily reported significant improvements in workplace and household productivity after 3 and 6 months of treatment compared with productivity prior to treatment and compared with women given placebo. 


\section{Introduction}

Endometriosis is a common gynecological disease in which endometrial-like tissue grows outside of the uterus in an estrogen-dependent manner [1]. Ectopic endometrial growths are associated with a chronic inflammatory state that promotes disease progression [2]. The condition is found in women of reproductive age, with the highest prevalence in women aged 35-44 years [3]. It is estimated that endometriosis affects approximately $10 \%$ of women worldwide [4], with a recent survey reporting a $6.1 \%$ prevalence among women aged 18-49 years in the United States of America (USA) [2]. The most prominent symptom of endometriosis is pain, which can include dysmenorrhea, chronic pelvic pain, lower back pain, dyspareunia, pain at ovulation, dyschezia, and dysuria; women with endometriosis also experience menorrhagia, fatigue, and infertility [5-7].

Many studies have found that endometriosis has a substantial negative impact on women's health-related quality of life (HRQOL) [7-11]. The chronic nature of endometriosis and the prominence of pain symptoms considerably affect all aspects of women's social, emotional, and physical well-being [7-9, 11]. Women with endometriosis report physical limitations such as impaired mobility, reduced energy, and difficulties performing daily activities and self-care [11, 12]. Furthermore, women commonly report experiencing anxiety/stress, depression, and negative impacts on intimate relationships and reproductive planning [8, 11-13]. A large, cross-sectional study of women with endometriosis in the USA found that the severity and number of symptoms was inversely correlated with HRQOL [2]. In particular, pelvic pain/cramping during menstrual periods, general abdominal pain, irregular periods, and dyspareunia had the most significant negative impacts on HRQOL [2].

In addition to the established negative effects of endometriosis on mental and physical well-being, several studies have documented the profound impact of endometriosis-associated symptoms on both employment-based and household productivity [9-11, 13, 14]. Missed time from work (absenteeism) and reduced effectiveness while at work (presenteeism) comprise overall workplace productivity loss. Women with endometriosis reportedly miss, on average, 3-13\% of work time owing to absenteeism and lose 14-65\% of productive work time owing to endometriosis symptoms, especially pain $[10,11,14]$. Reduced productivity in the household has also been reported; in one study, up to $79 \%$ of women reported significant impairments in performing household chores [13]. Moreover, a populationbased survey of women with endometriosis demonstrated a correlation between symptom severity and loss of productivity in the workplace and the household. Loss of workplace productivity in women with endometriosis carries a substantial societal economic burden. In the USA, the indirect cost of workplace absenteeism and short- and long-term disability in women with endometriosis was estimated to be US\$2132 per patient per year, which does not factor in losses due to presenteeism [15]. A 2011 study in the USA estimated that monetary losses due to presenteeism were fivefold higher than those due to absenteeism, with approximately US $\$ 250$ lost per week per patient from presenteeism and US\$50 lost per week per patient from absenteeism [10].

Given the substantial burden that endometriosis symptoms place on women's HRQOL and the high indirect costs to society, management strategies are needed to mitigate productivity loss associated with endometriosis. Estrogen is central to the pathophysiology of endometriosis, promoting endometrial tissue growth and inflammation [1]. Therefore, reduced estrogen can alleviate endometriosis-related symptoms, including pain [16]. Elagolix is an oral gonadotropinreleasing hormone receptor antagonist that results in dosedependent suppression of gonadotropins and ovarian sex steroids [17, 18]. Two phase III trials (ELARIS EM-I and ELARIS EM-II) have demonstrated that elagolix $150 \mathrm{mg}$ once daily (QD) and $200 \mathrm{mg}$ twice daily (BID) improves dysmenorrhea and nonmenstrual pelvic pain in women with moderate to severe pain associated with endometriosis after 3 and 6 months of treatment [18]. This post hoc analysis was conducted on data pooled from the ELARIS EM-I and EM-II trials to evaluate the impact of elagolix on workplace and household productivity in a large population of women with moderate to severe endometriosis-associated pain.

\section{Methods}

\subsection{Study Design and Participants}

The data for these analyses were pooled from two international phase III trials (ELARIS EM-I [NCT01620528] and ELARIS EM-II [NCT01931670]) for which the study designs and participant recruitment have been previously described [18]. Briefly, both were randomized, double-blind, multicenter, placebo-controlled studies that evaluated the efficacy and safety of two doses of elagolix (150 mg QD or $200 \mathrm{mg}$ BID) versus placebo in premenopausal women with moderate to severe endometriosis-associated pain [18]. Women aged 18-49 years who received a surgical diagnosis of endometriosis in the previous 10 years and who had moderate to severe endometriosis-associated pain were included in these studies. Women with a clinically significant gynecologic or chronic pain condition unrelated to endometriosis were excluded.

Women were randomly assigned 2:2:3 to receive elagolix $150 \mathrm{mg}$ QD, $200 \mathrm{mg}$ BID, or placebo for 6 months, 
with a 12-month follow-up period, or optional enrollment in a 6-month open-label extension period. The primary endpoints in both studies were the proportion of women with a clinical response (clinically meaningful reduction in pain score and decreased or stable use of analgesics) for dysmenorrhea and nonmenstrual pelvic pain at 3 months [18]. Patient-reported outcomes were measured as secondary outcomes in these studies. The present study evaluated change from baseline to each visit during the 6-month treatment period (Months 3 and 6) in workplace and household productivity per the Health-Related Productivity Questionnaire (HRPQ) [19]. Outcomes of patients in the extension period were not evaluated in this analysis.

\subsection{Health-Related Productivity Questionnaire (HRPQ)}

This post hoc analysis was performed to evaluate data from the administered HRPQ at baseline and during Months 3 and 6 of treatment. The HRPQ is a 9-item questionnaire that assesses a patient's ability to perform employmentbased work and daily activities in the home, measuring absenteeism (work time missed) and presenteeism (reduced work effectiveness) because of endometriosis in the workplace and in the household [19]. Patients who were employed part- or full-time and had scheduled work hours in the week prior to survey completion were eligible to respond to questions regarding workplace productivity. All patients with planned household work hours in the week prior to taking the survey, regardless of employment status, could answer questions related to household productivity. Respondents were asked to report scheduled employment-based work hours and planned household work hours in the previous week, which included household activities such as cooking, cleaning, gardening, and repairs.

The HRPQ was modified to be specific to endometriosis, asking study participants, "Did endometriosis-associated pain or its treatment(s) keep you from working any of your scheduled hours during the last week?" If the answer was "Yes," respondents gave the number of hours missed. To assess workplace presenteeism, study participants were asked, "For the hours that you did work during the past week, how did endometriosis-associated pain or its treatment(s) impact your work output?" Response options were on a $0-100 \%$ scale in which $0 \%$ indicated that endometriosis-associated pain/treatment had no impact on how much was accomplished and 100\% indicated that endometriosis-associated pain/treatment prevented the respondent from accomplishing anything. The hours lost to presenteeism were then calculated as:
Presenteeism $=$ actual hours worked

$$
\times(\% \text { impact on work output/100). }
$$

The same questions were asked regarding the impact of endometriosis-associated pain or treatment on household productivity due to absenteeism and presenteeism. Patient responses were used to calculate the number of employmentbased and household work hours spent away from workplace/ household work (absenteeism), productive hours lost while working in the workplace/household (presenteeism), and total hours lost owing to absenteeism and presenteeism in the workplace/household. The percentages of scheduled employmentbased work hours and planned household work hours lost due to absenteeism, presenteeism, and in total, were calculated.

\subsection{Statistical Analyses}

Analyses were performed with SAS version 9.3 (SAS Institute, Cary, NC, USA) using the UNIX operating system. The data were analyzed as observed, without imputation for missing responses. The patients included in these analyses were those who were randomized and received at least one dose of placebo or elagolix (modified intent-totreat population). Patients who responded to the question of absent and worked hours in the workplace at baseline were included in workplace productivity analyses. Patients who reported having planned household work hours in the week prior to taking the survey were included in household productivity analyses. Least squares (LS) means were calculated for the number and percentage of hours lost to absenteeism, presenteeism, and in total at baseline. Productivity gains at Months 3 and 6 of treatment were calculated as $-1 \times$ LS mean change from baseline in productive hours or the percentage of planned/scheduled productive hours. Differences between elagolix dose groups and placebo at baseline were assessed using an analysis of variance (ANOVA) model with treatment as the main effect. At Months 3 and 6 of treatment, differences between elagolix dose groups and placebo were assessed using an analysis of covariance (ANCOVA) model with treatment as the main effect and baseline productivity loss as a covariate. The corresponding $95 \%$ confidence intervals (CIs) and $p$ values were calculated. $P<0.05$ was considered statistically significant.

\section{Results}

\subsection{Baseline Characteristics}

A total of 1270 women with moderate to severe endometriosis-associated pain responded to workplace productivity 
questions in this analysis, including 556 treated with placebo, 359 treated with elagolix $150 \mathrm{mg}$ QD, and 355 treated with elagolix $200 \mathrm{mg}$ BID, representing $75 \%$ of all randomized patients. The baseline demographics and clinical characteristics for these patients were similar between treatment groups (Table 1). The average patient age was approximately 32 years in all treatment arms, and scores were very similar or the same among groups for dysmenorrhea (2.1 [SD 0.5]), nonmenstrual pelvic pain (1.5-1.6 [SD 0.5]), and dyspareunia (1.5 [SD 0.8-0.9]).

At baseline, mean total hours of workplace productivity lost in the previous week $(16 \mathrm{~h})$ was similar among groups, with $3 \mathrm{~h}$ lost owing to absenteeism and $13 \mathrm{~h}$ lost owing to presenteeism. Across treatment arms, women reported an average loss of $45 \%$ of scheduled workplace productivity, with fourfold greater loss due to presenteeism than the loss due to absenteeism. Household productivity data were available for 1565 women who reported having planned household work hours in the week prior to taking the survey, representing approximately $93 \%$ of all randomized patients $(681$ received placebo, 437 received elagolix $150 \mathrm{mg}$ QD, and 447 received elagolix $200 \mathrm{mg}$ BID; Table 1). On average, $8.3 \mathrm{~h}$ of household productivity were lost at baseline across treatment groups, with $4.7 \mathrm{~h}$ lost due to absenteeism and $3.6 \mathrm{~h}$ lost due to presenteeism. Women lost $64 \%$ of planned household productivity hours at baseline, with $38 \%$ loss due to absenteeism and $26 \%$ loss due to presenteeism. Therefore, absenteeism accounted for $60 \%$ of the total planned household productivity lost, whereas presenteeism accounted for $40 \%$ of planned household productivity lost.

Table 1 Baseline characteristics of patients in analysis (modified intent-to-treat) ${ }^{\mathrm{a}}$

\begin{tabular}{|c|c|c|c|c|c|c|}
\hline Characteristic & $N$ & Placebo & $N$ & Elagolix $150 \mathrm{mg}$ QD & $N$ & Elagolix $200 \mathrm{mg}$ BID \\
\hline Age, years, mean $\pm \mathrm{SD}$ & 556 & $32.7 \pm 6.5$ & 359 & $32.4 \pm 6.4$ & 355 & $32.4 \pm 6.6$ \\
\hline $\mathrm{BMI}, \mathrm{kg} / \mathrm{m}^{2}$, mean $\pm \mathrm{SD}$ & 553 & $27.5 \pm 6.1$ & 358 & $27.7 \pm 6.6$ & 351 & $27.3 \pm 6.4$ \\
\hline $\begin{array}{l}\text { Dysmenorrhea score, mean } \pm \text { SD } \\
(\text { scale } 0-3)^{b}\end{array}$ & 556 & $2.1 \pm 0.5$ & 359 & $2.1 \pm 0.5$ & 355 & $2.1 \pm 0.5$ \\
\hline Nonmenstrual pelvic pain score, mean $\pm \operatorname{SD}(\text { scale } 0-3)^{\mathrm{b}}$ & 556 & $1.6 \pm 0.5$ & 359 & $1.6 \pm 0.5$ & 355 & $1.5 \pm 0.5$ \\
\hline Dyspareunia score, mean \pm SD $(\text { scale } 0-3, \text { or N/A })^{c}$ & 456 & $1.5 \pm 0.8$ & 294 & $1.5 \pm 0.9$ & 289 & $1.5 \pm 0.9$ \\
\hline $\begin{array}{l}\text { Hours of scheduled employment-based work in previous week, LS } \\
\text { mean } \pm \mathrm{SE}\end{array}$ & 556 & $33.0 \pm 0.5$ & 359 & $34.2 \pm 0.7$ & 355 & $33.0 \pm 0.7$ \\
\hline \multicolumn{7}{|l|}{ Hours of workplace productivity lost, LS mean \pm SE } \\
\hline Total hours lost & 556 & $15.7 \pm 0.5$ & 359 & $16.2 \pm 0.6$ & 355 & $16.0 \pm 0.6$ \\
\hline Absenteeism & 556 & $3.2 \pm 0.2$ & 359 & $3.0 \pm 0.3$ & 355 & $3.0 \pm 0.3$ \\
\hline Presenteeism & 552 & $12.6 \pm 0.4$ & 356 & $13.4 \pm 0.5$ & 350 & $13.2 \pm 0.5$ \\
\hline \multicolumn{7}{|l|}{ Percentage of workplace productivity lost, LS mean \pm SE } \\
\hline Total percentage of hours lost & 556 & $44.0 \pm 1.2$ & 359 & $44.5 \pm 1.4$ & 355 & $45.3 \pm 1.5$ \\
\hline Absenteeism & 556 & $9.8 \pm 0.7$ & 359 & $8.3 \pm 0.9$ & 355 & $9.6 \pm 0.9$ \\
\hline Presenteeism & 552 & $34.4 \pm 1.0$ & 356 & $36.6 \pm 1.2$ & 350 & $36.3 \pm 1.3$ \\
\hline Hours of planned household work in previous week, LS mean \pm SE & 681 & $7.7 \pm 0.3$ & 437 & $8.1 \pm 0.4$ & 447 & $7.9 \pm 0.4$ \\
\hline \multicolumn{7}{|l|}{ Hours of household productivity lost, LS mean \pm SE } \\
\hline Total hours lost & 681 & $8.3 \pm 0.3$ & 437 & $8.6 \pm 0.4$ & 447 & $8.1 \pm 0.4$ \\
\hline Absenteeism & 681 & $4.7 \pm 0.2$ & 437 & $4.8 \pm 0.3$ & 447 & $4.7 \pm 0.3$ \\
\hline Presenteeism & 679 & $3.6 \pm 0.2$ & 435 & $3.8 \pm 0.2$ & 443 & $3.4 \pm 0.2$ \\
\hline \multicolumn{7}{|l|}{ Percentage of household productivity lost, LS mean \pm SE } \\
\hline Total percentage of hours lost & 681 & $65.6 \pm 1.1$ & 437 & $63.4 \pm 1.4$ & 447 & $64.4 \pm 1.4$ \\
\hline Absenteeism & 681 & $39.1 \pm 1.1$ & 437 & $37.4 \pm 1.4$ & 447 & $38.7 \pm 1.4$ \\
\hline Presenteeism & 679 & $26.6 \pm 0.7$ & 435 & $26.2 \pm 0.9$ & 443 & $25.9 \pm 0.9$ \\
\hline
\end{tabular}

${ }^{a}$ Women employed full- or part-time with scheduled work hours in the week prior to taking the survey were included in workplace productivity analyses $(n=1270)$; women with planned household work hours in the week prior to taking the survey, regardless of employment status, were included in household productivity analyses $(n=1565)$

${ }^{\mathrm{b}}$ Pain responses were none $=0$, mild $=1$, moderate $=2$, and severe $=3$; group mean is based on the daily average score over the 35-day interval ${ }^{c}$ Pain responses were none $=0$, mild $=1$, moderate $=2$, severe $=3$, and not applicable; group mean is based on the daily average score over the 35-day interval. Subjects responding 'not applicable' for the entire 35-day interval are excluded for a total of 1384 subjects with baseline dyspareunia pain responses

$B I D$ twice daily, $B M I$ body mass index, $L S$ least squares, N/A not applicable, $Q D$ once daily, $S D$ standard deviation, $S E$ standard error 


\subsection{Impact of Elagolix on Employment-Based Productivity}

Women given placebo gained a total of 5.9 productive workplace hours per week by Month 3 and 6.9 h by Month 6 (Fig. 1). Both doses of elagolix were associated with significantly greater improvements than placebo in productive workplace hours per week at Months 3 and 6 of treatment (Fig. 1). At Month 3, women gained 2.4 productive workplace hours (95\% CI $0.9-3.9 ; p=0.002)$ and 4.7 productive workplace hours (95\% CI 3.6-6.2; $p<0.001$ ) per week relative to placebo with elagolix $150 \mathrm{mg}$ QD and $200 \mathrm{mg}$ BID, respectively. At Month 6, workplace productivity increased by $1.7 \mathrm{~h}(95 \% \mathrm{CI} 0.1-3.4 ; p=0.041)$ and $5.4 \mathrm{~h}(95 \% \mathrm{CI}$ $3.7-7.1 ; p<0.001)$ per week relative to placebo with elagolix $150 \mathrm{mg}$ QD and $200 \mathrm{mg}$ BID, respectively. Gains in productive workplace hours owing to reduced absenteeism were significantly higher than placebo for both doses at Month 3 and only the 200-mg dose at Month 6. Reduced presenteeism accounted for $63-71 \%$ of total gains relative to placebo in productive workplace hours. At Month 3, elagolix $150 \mathrm{mg}$ QD and $200 \mathrm{mg}$ BID were associated with 1.5-h (95\% CI $0.2-2.8 ; p=0.022)$ and 3.1 -h $(95 \%$ CI $1.8-4.3 ; p<0.001)$ gains, respectively, relative to placebo in weekly productive workplace hours due to reduced presenteeism. By Month 6, women treated with elagolix $200 \mathrm{mg}$ BID reported a 3.8-h (95\% CI 2.4-5.2; $p<0.001)$ increase relative to placebo in productive workplace hours due to reduced presenteeism.
Treatment with elagolix $150 \mathrm{mg}$ QD or $200 \mathrm{mg}$ BID was associated with significant gains over placebo in the percentage of scheduled employment-based work hours actually worked (Fig. 2). At Month 3, women treated with elagolix $150 \mathrm{mg}$ QD and $200 \mathrm{mg}$ BID had gains of 6.6\% (95\% CI 2.7-10.4) and 11.6\% (95\% CI 7.7-15.5), respectively (both $p<0.001$ ), of scheduled employment-based work relative to placebo. At Month 6, elagolix $200 \mathrm{mg}$ BID was associated with a gain of $14.6 \%$ of scheduled work relative to placebo (95\% CI $10.0-19.1 ; p<0.001)$, versus a $5.2 \%$ gain with elagolix $150 \mathrm{mg}$ QD (95\% CI 0.7-9.7; $p=0.022$ ). Reduced absenteeism significantly contributed to productivity gains per scheduled work with both doses of elagolix at Month 3 and with elagolix $200 \mathrm{mg}$ BID at Month 6. Decreased presenteeism accounted for 59-66\% of total workplace gains relative to placebo in scheduled work at Months 3 and 6 of elagolix treatment. Gains in the percentage of scheduled workplace hours from decreased presenteeism were significant with elagolix $150 \mathrm{mg}$ QD and $200 \mathrm{mg}$ BID at Month 3 $(p=0.021$ and $p<0.001$, respectively) and elagolix $200 \mathrm{mg}$ BID at Month $6(p<0.001)$.

\subsection{Impact of Elagolix on Household Productivity}

In addition to improved workplace productivity, women given placebo also gained a total of 3.0 productive household hours per week by Month 3 and 3.1 h by Month 6 (Fig. 3). Both elagolix doses were associated with improvements

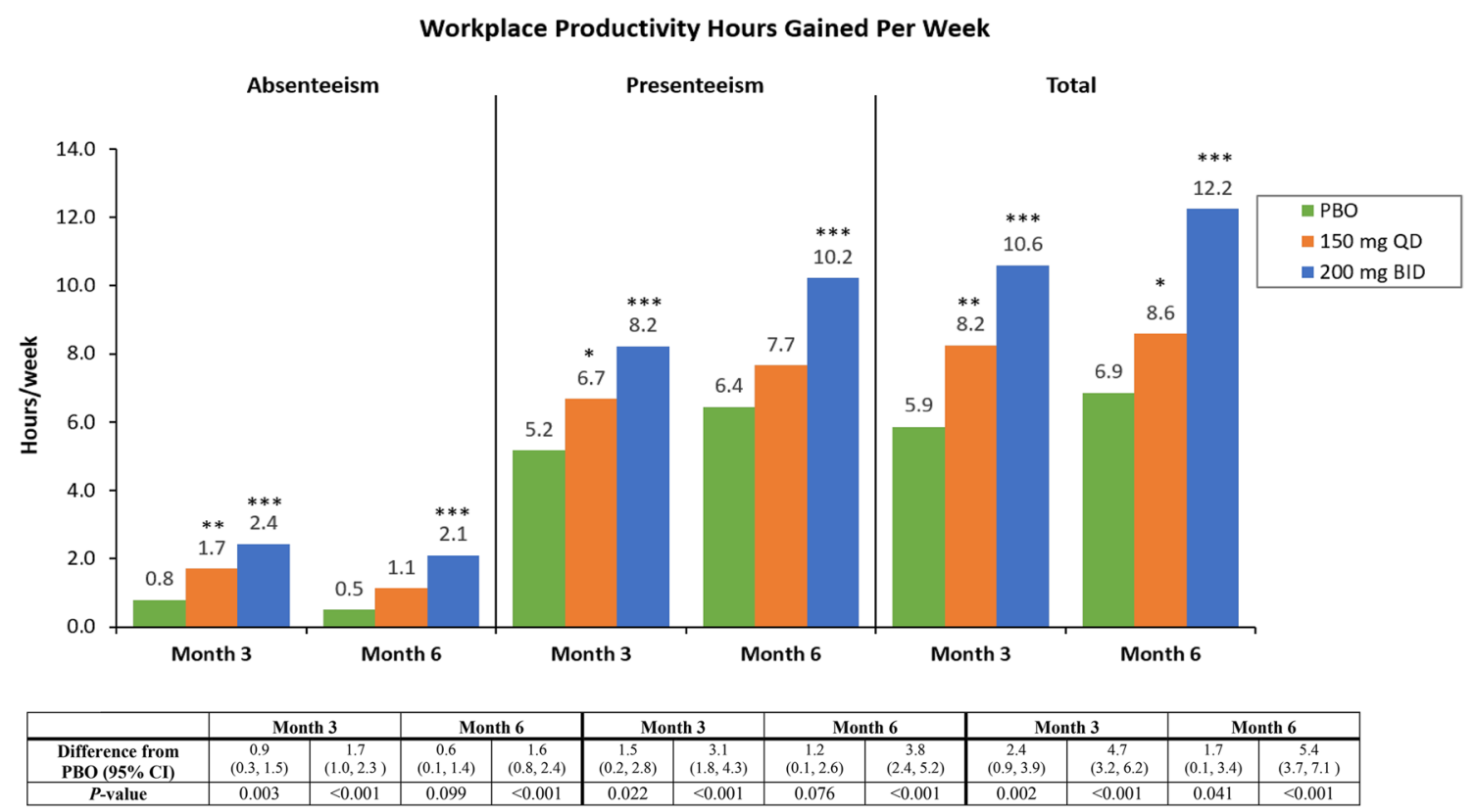

Fig. 1 Gains in productive workplace hours per week at Months 3 and 6 of treatment. Mean hours gained in workplace productivity from baseline, defined as $-1 \times \mathrm{LS}$ mean change from baseline in hours of workplace productivity lost due to absenteeism, presentee- ism, and total hours lost (absenteeism + presenteeism). $* * * p<0.001$; ${ }^{* *} p<0.01 ;{ }^{*} p<0.05$. BID twice daily, $C I$ confidence interval, $L S$ least squares, $P B O$ placebo, $Q D$ once daily 


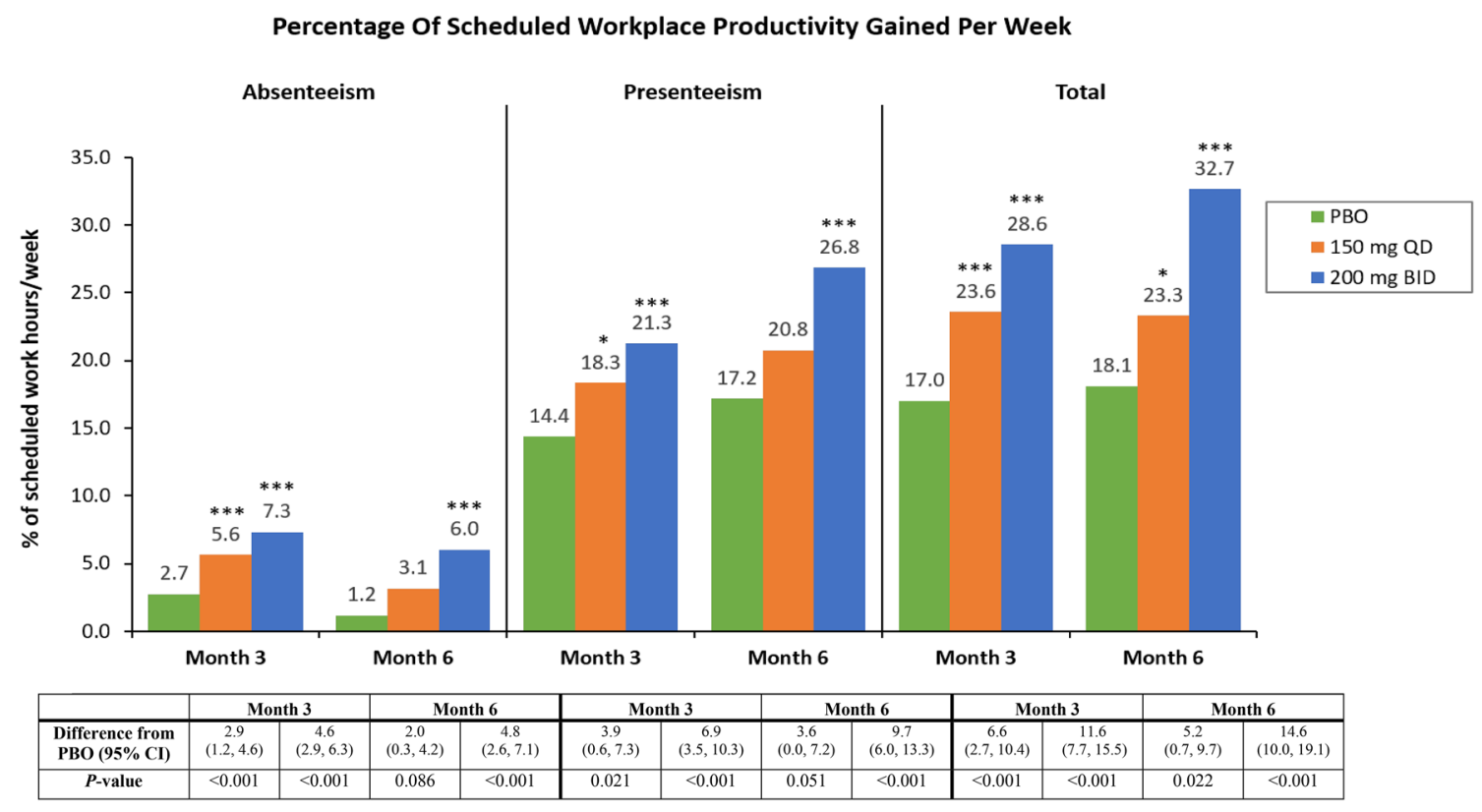

Fig. 2 Gains in percentage of scheduled workplace productivity per week at Months 3 and 6 of treatment. Mean gains in the percentage of scheduled workplace hours worked, defined as $-1 \times$ LS mean change from baseline in percentage of scheduled workplace hours lost due to absenteeism, presenteeism, and total (absenteeism + presenteeism). $*^{* *} p<0.001 ; * * p<0.01 ; * p<0.05$. BID twice daily, $C I$ confidence interval, $L S$ least squares, $P B O$ placebo, $Q D$ once daily

CI 3.5-14.1; $p=0.001)$, while elagolix $200 \mathrm{mg}$ BID was associated with a gain of $20.4 \%$ relative to placebo (95\% CI 15.1-25.6; $p<0.001)$. Reduced absenteeism at Month 6 corresponded to a gain of $4.7 \%$ (95\% CI $0.6-8.8 ; p=0.024)$ of planned household productivity relative to placebo with elagolix $150 \mathrm{mg}$ QD and 13.0\% (95\% CI 8.9-17.0; $p<0.001$ ) with elagolix $200 \mathrm{mg}$ BID. Decreased presenteeism accounted for a gain of $4.7 \%$ of planned household productivity relative to placebo $(95 \% \mathrm{CI} 1.7-7.6 ; p=0.002)$ in women treated with elagolix $150 \mathrm{mg}$ QD and $7.5 \%$ (95\% CI $4.6-10.4 ; p<0.001)$ in women treated with elagolix $200 \mathrm{mg}$ BID at Month 6. treated for 3 months with both elagolix doses had significant increases in productive household hours from reduced absenteeism $(p<0.001)$. At Month 6, productive household hours increased by 1.0 (95\% CI 0.4-1.7) and 2.0 (95\% CI 1.4-2.7) hours relative to placebo due to reduced absenteeism with elagolix $150 \mathrm{mg}$ QD $(p=0.003)$ and $200 \mathrm{mg}$ BID $(p<0.001)$, respectively. Gains in household productivity due to reduced presenteeism were less pronounced but still significant for women treated with elagolix $200 \mathrm{mg}$ BID at Month $3(p=0.049)$ and both doses at Month 6 (150 mg QD, $p=0.016 ; 200 \mathrm{mg} \mathrm{BID}, p<0.001)$.

There were also large gains in the percentage of household productivity per planned household work at both timepoints with both elagolix doses (Fig. 4). At Month 6, elagolix $150 \mathrm{mg}$ QD was associated with a gain of $8.8 \%$ of planned household productivity relative to placebo $(95 \%$

\section{Discussion}

This analysis of pooled data from two large, placebo-controlled trials [18] demonstrates that treatment with elagolix significantly improves productivity in both the workplace and household among women with moderate to severe endometriosis-associated pain. Previous research has established a clear negative impact of endometriosis-associated pain on women's HRQOL and productivity [7-11]. Importantly, this study is among the first to demonstrate that a treatment which effectively manages endometriosis pain also positively impacts patients' ability to productively participate in the workforce and perform household tasks. 


\section{Household Productivity Hours Gained Per Week}

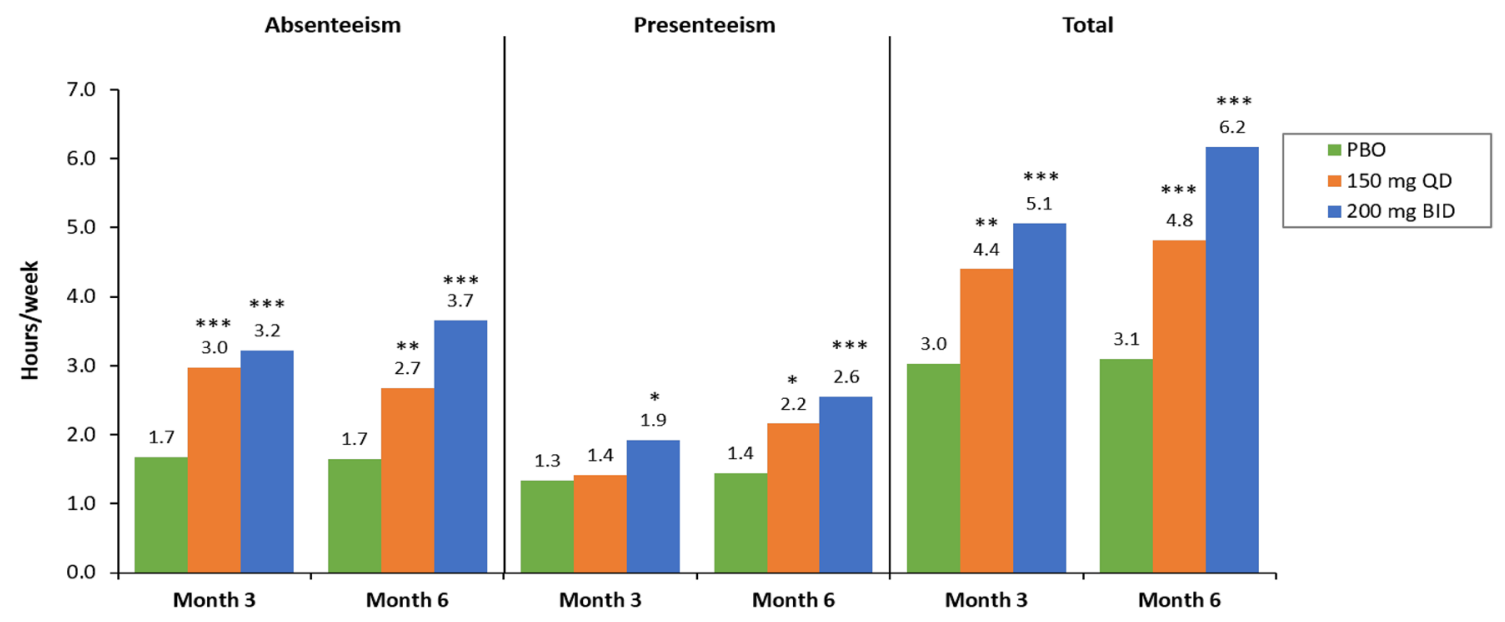

\begin{tabular}{|c|c|c|c|c|c|c|c|c|c|c|c|c|}
\hline & \multicolumn{2}{|c|}{ Month 3 } & \multicolumn{2}{c|}{ Month 6 } & \multicolumn{2}{c|}{ Month 3 } & \multicolumn{2}{c|}{ Month 6 } & \multicolumn{3}{c|}{ Month 3 } & \multicolumn{2}{c|}{ Month 6 } \\
\hline $\begin{array}{c}\text { Difference from } \\
\text { PBO (95\% CI) }\end{array}$ & $1.3 .7 .7 .9)$ & $(0.9,2.2)$ & $(0.4,1.7)$ & $(1.4,2.7)$ & $(-0.5,0.7)$ & $(0.0,1.2)$ & $(0.1,1.3)$ & $(0.5,1.7)$ & $(0.5,2.3)$ & $(1.1,3.0)$ & $(0.7,2.7)$ & $(2.1,4.0)$ \\
\hline P-value & $<0.001$ & $<0.001$ & 0.003 & $<0.001$ & 0.764 & 0.049 & 0.016 & $<0.001$ & 0.004 & $<0.001$ & $<0.001$ & $<0.001$ \\
\hline
\end{tabular}

Fig. 3 Gains in hours of household productivity per week at Months 3 and 6 of treatment. Mean hours gained in household productivity, defined as $-1 \times$ LS mean change from baseline in hours of household productivity lost due to absenteeism, presenteeism, and total (absen- teeism + presenteeism). $\quad * * * p<0.001 ; \quad * * p<0.01 ; * p<0.05 . \quad B I D$ twice daily, $C I$ confidence interval, $L S$ least squares, $P B O$ placebo, $Q D$ once daily

Percentage Of Planned Household Productivity Gained Per Week

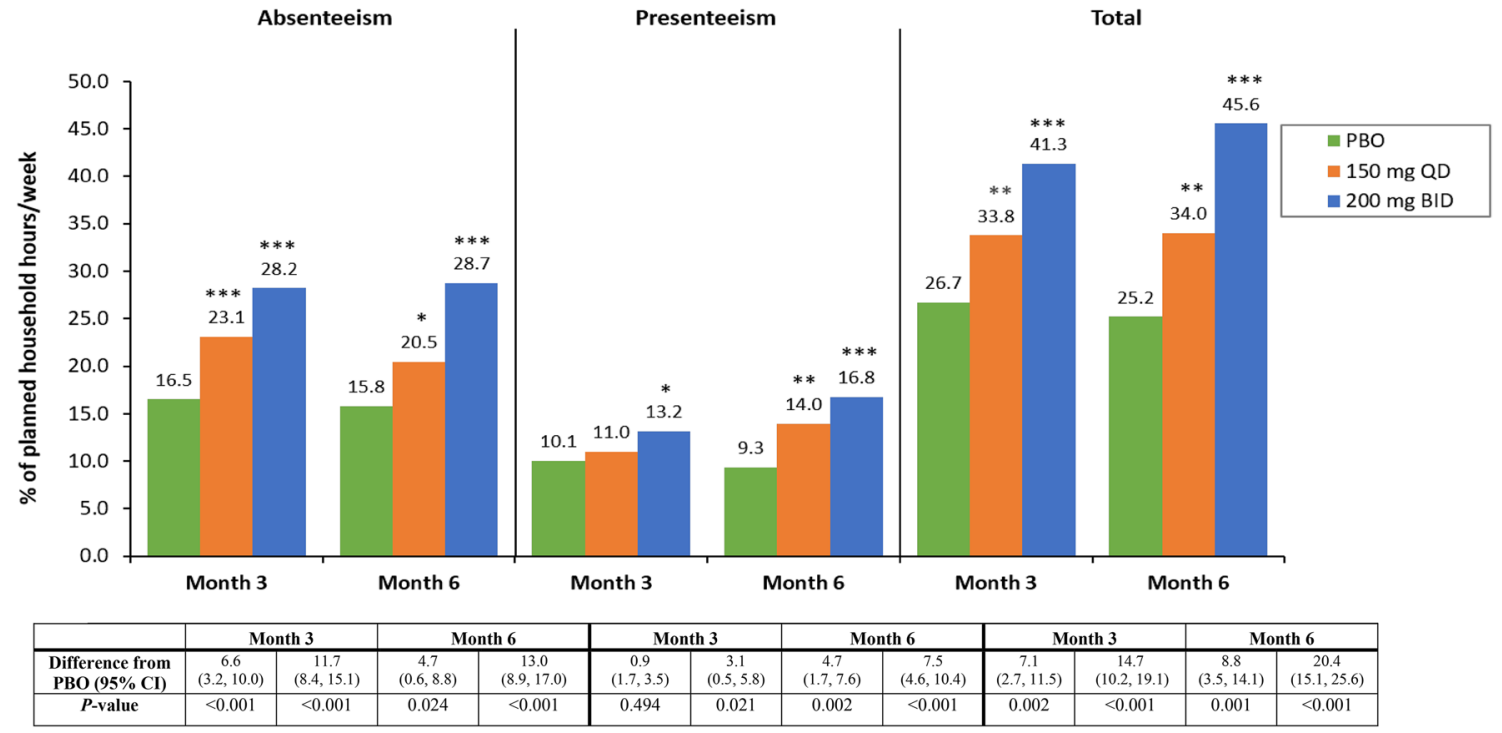

Fig. 4 Gains in percentage of planned household productivity per week at Months 3 and 6 of treatment. Mean gains in the percentage of planned household work hours worked, defined as $-1 \times$ LS mean change from baseline in percentage of planned household work hours

In the current study, women with moderate to severe endometriosis-associated pain experienced a significant loss in workplace and household productivity prior to treatment that approached a total of $45 \%$ of scheduled lost due to absenteeism, presenteeism, and total (absenteeism + presenteeism). ${ }^{* * *} p<0.001 ; * * p<0.01 ;{ }^{*} p<0.05$. BID twice daily, $C I$ confidence interval, $L S$ least squares, $P B O$ placebo, $Q D$ once daily

employment-based work and $64 \%$ of planned household work. The impact of endometriosis on women's daily lives is underscored by the $20 \%$ greater loss in household productivity than workplace productivity. These results are generally 
consistent with previous studies that found substantial productivity impairments in women with endometriosis [10, $11,13,14]$. At baseline, women in this study lost an average of $16 \mathrm{~h}$ of workplace productivity and $8 \mathrm{~h}$ of household productivity per week. The magnitude of productivity losses reported here by women with endometriosis are greater than those reported by patients with other chronic pain conditions. Patients with rheumatoid arthritis reported a total of $29 \%$ workplace productivity loss and $41 \%$ impairment of daily activities, and patients with inflammatory bowel disease reported an overall $21 \%$ work productivity loss and $30 \%$ activity impairment [20]. At baseline, common pain conditions, such as headache and back pain, were associated with 3.5 and $5.3 \mathrm{~h}$ of productive work time lost per week, respectively [21], significantly less work time lost than reported here. Impaired productivity not only stems from pain-related endometriosis symptoms, but also from moderate/severe symptoms such as fatigue, heavy menstrual bleeding, spotting/bleeding during periods, irregular periods, and bloating. Moreover, women with endometriosis have a significantly higher incidence of comorbid conditions [22]. The presence of comorbid ovarian cysts, depression, and hypertension were found to be strong predictors for workplace and household productivity losses, suggesting that comorbid conditions may increase productivity loss.

Treatment with elagolix was associated with significant gains in productive workplace hours, equivalent to restoring 1-1.5 work days per week, versus 0.75 work days per week with placebo. The impact of endometriosis on women's participation in the workforce may be underappreciated if based solely on time absent from work. Decreased efficiency and productivity while at work due to endometriosis-related symptoms (presenteeism) heavily contributes to productivity losses. In the current study, workplace productivity losses at baseline due to presenteeism were approximately 4-4.5 times as large as losses due to absenteeism. Previous studies in women with endometriosis have documented 2.3 [10], 2.7 [11], and 4.8 [14] times greater work productivity losses from presenteeism. These findings suggest that women with endometriosis are present at work despite experiencing symptoms that limit their ability to effectively perform work $[10,11$, 14]. Symptoms that predict poor performance at work include dysmenorrhea, nonmenstrual pelvic pain, dyspareunia, irregular periods, abdominal pain, incapacitating pain, and depression [13, 14]. Unlike workplace productivity losses, household productivity losses at baseline due to absenteeism and presenteeism were similar, with approximately 1.4 times greater productivity loss due to absenteeism than presenteeism. The ratio of household absenteeism to presenteeism reported in the current study is similar to that reported previously (ratio 1.1) in women in the USA with endometriosis.

Importantly, women treated with elagolix $150 \mathrm{mg}$ QD and $200 \mathrm{mg}$ BID reported substantial improvements in workplace and household productivity, including productivity gains due to reduced absenteeism and presenteeism, compared with women given placebo. Significant gains in both workplace and household productivity were reported by Month 3 of treatment with elagolix and persisted or were greater by Month 6. Generally, elagolix $200 \mathrm{mg}$ BID was associated with greater improvements in productivity than elagolix $150 \mathrm{mg}$ QD. By Month 6, treatment with elagolix $200 \mathrm{mg}$ BID was associated with a $15 \%$ increase in workplace productivity per scheduled work hours and a $20 \%$ increase in household productivity per planned household work hours, relative to placebo. Taken together, these patient-reported results suggest that elagolix $150 \mathrm{mg}$ QD and $200 \mathrm{mg}$ BID effectively mitigate the negative impact of endometriosis symptoms on employment-based and household productivity.

Elagolix $150 \mathrm{mg}$ QD and $200 \mathrm{mg}$ BID reduce dysmenorrhea and nonmenstrual pelvic pain [18], symptoms which, when moderate or severe, are strongly correlated with greater productivity losses [14]. In ELARIS EM-I and EM-II studies, $42.1-46.4 \%$ of women taking elagolix $150 \mathrm{mg}$ QD and $72.4-76.9 \%$ of women taking elagolix $200 \mathrm{mg}$ BID experienced clinically meaningful pain reduction and decreased or stable use of rescue analgesics for dysmenorrhea over 6 months of treatment, compared with 19.6-25.4\% of women receiving placebo [18]. Women receiving both elagolix doses also experienced pain reduction and reduced analgesic use for nonmenstrual pelvic pain (45.7-51.6\% with elagolix $150 \mathrm{mg}$ QD; 54.5-62.2\% with elagolix $200 \mathrm{mg}$ BID; 34.9-40.6\% with placebo) [18]. The current analysis was not designed to correlate pain improvement scores with productivity improvements, and it is therefore possible that the impact of elagolix on non-pain symptoms may also contribute to improvements in productivity. Beyond pain, fatigue is a highly prevalent symptom of endometriosis, which may be intense and bothersome $[8,23,24]$ and has been associated with impairments in workplace, household, and social activities [14, 25]. Treatment with elagolix $150 \mathrm{mg}$ QD and $200 \mathrm{mg}$ BID significantly improves fatigue at both 3 and 6 months of treatment in women with moderate to severe endometriosis-associated pain [24]. Fatigue and endometriosis-associated pain symptoms are interrelated, with individual pain symptoms (dysmenorrhea, nonmenstrual pelvic pain, dyspareunia) increasing fatigue [24]. Thus, improvements in endometriosis-associated pain and fatigue from elagolix treatment may both play a role in improving productivity.

The present study is the first to assess whether an endometriosis treatment can address the substantial productivity impairments women experience, but the study has some limitations. As previously discussed, the HRPQ is a selfreported instrument and therefore results may suffer from recall bias and reporting errors. For example, reporting on 
planned household chore hours for the previous week relied on a priori prediction of the number of hours respondents intended to spend on household chores, whereas scheduled work hours for the previous week was based on empirical knowledge. The bias may be mitigated given that the recall period was only 1 week. The HRPQ has good construct and criterion validity but requires further research and reliability testing [19]. The productivity outcomes reported here were from a large cohort of patients in a randomized, wellcontrolled clinical trial, and therefore may not be representative of the general population of women with endometriosis. Furthermore, the study was not designed to compare outcomes between the two doses of elagolix, only to compare outcomes against placebo. While many sociodemographic and health characteristics can influence a person's participation in the workforce, this study did not evaluate the impact of these factors on productivity outcomes. Although women treated with placebo reported higher-than-expected productivity improvements, the productivity gains associated with elagolix treatment remained significantly higher than placebo (1.2- to 2-fold higher). It is possible that the effects observed with placebo were related to use of analgesic rescue medication during the trial.

Despite these limitations, the results reported herein provide needed insight as to the management of workplace and household productivity impairment in women with moderate to severe endometriosis-associated pain. Moreover, these data are important to dispel negative perceptions of women with endometriosis, such as exaggeration of symptoms [26] and using endometriosis as an excuse to avoid work. The data in this study argue against the idea that women with endometriosis are simply avoiding work and further reinforce the pervasive and debilitating nature of endometriosis. Overall, elagolix appears to reverse endometriosis-related productivity losses, enhancing women's HRQOL and reducing the indirect costs of endometriosis.

\section{Conclusion}

Women with moderate to severe endometriosis-associated pain experience substantial impairments in workplace and household productivity, both in work hours missed and reduced work effectiveness due to symptoms. Treatment with elagolix $150 \mathrm{mg}$ QD or $200 \mathrm{mg}$ BID significantly improves workplace and household productivity among women with moderate to severe endometriosis-associated pain. Further research is needed to assess long-term treatment with elagolix as a management strategy for endometriosis-associated pain and concomitant productivity loss.

Data Sharing Statement AbbVie is committed to responsible data sharing regarding the clinical trials we sponsor.
Access is provided to anonymized, patient- and trial-level data (analysis data sets), as well as other information (e.g., protocols and Clinical Study Reports) from AbbVie-sponsored phase II-IV global interventional clinical trials conducted in patients (completed as of May 2004, for products and indications approved in either the United States or the European Union), as long as the trials are not part of an ongoing or planned regulatory submission). This includes requests for clinical trial data for unlicensed products and indications.

Access to this clinical trial data can be requested by any qualified researchers who engage in rigorous, independent scientific research, and will be provided following review and approval of a research proposal and Statistical Analysis Plan (SAP) and execution of a Data Sharing Agreement (DSA). Data requests can be submitted at any time and the data will be accessible for 12 months, with possible extensions considered. For more information on the process, or to submit a request, visit the following link: https://www. abbvie.com/our-science/clinical-trials/clinical-trials-dataand-information-sharing/data-and-information-sharingwith-qualified-researchers.html.

Acknowledgements This study was funded by AbbVie, Inc. AbbVie sponsored the study; contributed to the design; participated in collection, analysis, and interpretation of data; and in writing, reviewing, and approval of the final version. Medical writing assistance was provided by Emily Mercadante, $\mathrm{PhD}$, of JK Associates, Inc., a member of the Fishawack Group of Companies, Conshohocken, PA, USA, and was funded by AbbVie, Inc., North Chicago, IL, USA.

Authors' contributions All authors were responsible for the study's design and conception and for interpretation of data, drafting of the manuscript, and critical revision of the manuscript for important intellectual content. Statistical analyses were performed by A.M. Soliman and H.L. Palac. All authors read and approved the final manuscript.

\section{Compliance with Ethical Standards}

Funding This study was funded by AbbVie, North Chicago, IL, USA. AbbVie was involved in developing the study concept and participated in the analysis and interpretation of the data; preparation, review, and approval of the article; and decision to submit the article for publication.

Conflict of interest Eric S. Surrey is Medical Director at Colorado Center for Reproductive Medicine, has served in a consulting role for AbbVie and DOT Laboratories, received research grants from AbbVie, and served on the speaker bureau for AbbVie and Ferring Laboratories. Ahmed M. Soliman is an employee of, owns stock/stock options, and holds patents for AbbVie. Hannah L. Palac was an employee of AbbVie Inc. at the time the research was conducted. Sanjay K. Agarwal is Director of Fertility Services in the UC San Diego Department of Reproductive Medicine, Director of the UC San Diego Center for Endometriosis Research and Treatment, and has served in a consulting role on research for AbbVie Inc. and has received research support from AbbVie Inc. 
Ethical approval Shulman Associates Institutional Review Board (IRB) conducted the majority of the IRB approvals at each study center. The trial was conducted in accordance with the Declaration of Helsinki and International Conference on Harmonisation guidelines.

Informed consent Informed consent was obtained from all individuals participating in the study.

Open Access This article is distributed under the terms of the Creative Commons Attribution-NonCommercial 4.0 International License (http://creativecommons.org/licenses/by-nc/4.0/), which permits any noncommercial use, distribution, and reproduction in any medium, provided you give appropriate credit to the original author(s) and the source, provide a link to the Creative Commons license, and indicate if changes were made.

\section{References}

1. Reis FM, Petraglia F, Taylor RN. Endometriosis: hormone regulation and clinical consequences of chemotaxis and apoptosis. Hum Reprod Update. 2013;19(4):406-18. https://doi.org/10.1093/ humupd/dmt010.

2. Fuldeore MJ, Soliman AM. Prevalence and symptomatic burden of diagnosed endometriosis in the United States: national estimates from a cross-sectional survey of 59,411 women. Gynecol Obstet Invest. 2017;82(5):453-61. https://doi.org/10.1159/000452660.

3. Abbas S, Ihle P, Koster I, Schubert I. Prevalence and incidence of diagnosed endometriosis and risk of endometriosis in patients with endometriosis-related symptoms: findings from a statutory health insurance-based cohort in Germany. Eur J Obstet Gynecol Reprod Biol. 2012;160(1):79-83. https://doi.org/10.1016/j.ejogr b.2011.09.041.

4. Wheeler JM. Epidemiology of endometriosis-associated infertility. J Reprod Med. 1989;34(1):41-6.

5. Kennedy S, Bergqvist A, Chapron C, D'Hooghe T, Dunselman $\mathrm{G}$, Greb R, et al. ESHRE guideline for the diagnosis and treatment of endometriosis. Hum Reprod. 2005;20(10):2698-704. https://doi.org/10.1093/humrep/dei135.

6. Johnson NP, Hummelshoj L, World Endometriosis Society Montpellier Consortium. Consensus on current management of endometriosis. Hum Reprod. 2013;28(6):1552-68. https:// doi.org/10.1093/humrep/det050.

7. De Graaff AA, D'Hooghe TM, Dunselman GA, Dirksen CD, Hummelshoj L, WERF EndoCost Consortium, et al. The significant effect of endometriosis on physical, mental and social wellbeing: results from an international cross-sectional survey. Hum Reprod. 2013;28(10):2677-85. https://doi.org/10.1093/ humrep/det 284 .

8. Soliman AM, Coyne KS, Zaiser E, Castelli-Haley J, Fuldeore MJ. The burden of endometriosis symptoms on health-related quality of life in women in the United States: a cross-sectional study. J Psychosom Obstet Gynaecol. 2017;38(4):238-48. https ://doi.org/10.1080/0167482X.2017.1289512.

9. Culley L, Law C, Hudson N, Denny E, Mitchell H, Baumgarten $\mathrm{M}$, et al. The social and psychological impact of endometriosis on women's lives: a critical narrative review. Hum Reprod Update. 2013;19(6):625-39. https://doi.org/10.1093/humupd/ dmt027.

10. Nnoaham KE, Hummelshoj L, Webster P, d'Hooghe T, de Cicco Nardone F, de Cicco Nardone $\mathrm{C}$ et al. Impact of endometriosis on quality of life and work productivity: a multicenter study across ten countries. Fertil Steril. 2011;96(2):366-73 e8. https://doi. org/10.1016/j.fertnstert.2011.05.090.
11. Fourquet J, Baez L, Figueroa M, Iriarte RI, Flores I. Quantification of the impact of endometriosis symptoms on health-related quality of life and work productivity. Fertil Steril. 2011;96(1):107-12. https://doi.org/10.1016/j.fertnstert.2011.04.095.

12. Simoens S, Dunselman G, Dirksen C, Hummelshoj L, Bokor A, Brandes I, et al. The burden of endometriosis: costs and quality of life of women with endometriosis and treated in referral centres. Hum Reprod. 2012;27(5):1292-9. https://doi.org/10.1093/humre $\mathrm{p} / \mathrm{des} 073$.

13. Fourquet J, Gao X, Zavala D, Orengo JC, Abac S, Ruiz A, et al. Patients' report on how endometriosis affects health, work, and daily life. Fertil Steril. 2010;93(7):2424-8. https://doi. org/10.1016/j.fertnstert.2009.09.017.

14. Soliman AM, Coyne KS, Gries KS, Castelli-Haley J, Snabes MC, Surrey ES. The effect of endometriosis symptoms on absenteeism and presenteeism in the workplace and at home. J Manag Care Spec Pharm. 2017;23(7):745-54. https://doi.org/10.18553 /jmcp.2017.23.7.745.

15. Soliman AM, Surrey E, Bonafede M, Nelson JK, Castelli-Haley $\mathrm{J}$. Real-world evaluation of direct and indirect economic burden among endometriosis patients in the United States. Adv Ther. 2018;35(3):408-23. https://doi.org/10.1007/s12325-018-0667-3.

16. Barbieri RL. Hormone treatment of endometriosis: the estrogen threshold hypothesis. Am J Obstet Gynecol. 1992;166(2):740-5. https://doi.org/10.1016/0002-9378(92)91706-g.

17. Ng J, Chwalisz K, Carter DC, Klein CE. Dose-dependent suppression of gonadotropins and ovarian hormones by elagolix in healthy premenopausal women. J Clin Endocrinol Metab. 2017;102(5):1683-91. https://doi.org/10.1210/jc.2016-3845.

18. Taylor HS, Giudice LC, Lessey BA, Abrao MS, Kotarski J, Archer $\mathrm{DF}$, et al. Treatment of endometriosis-associated pain with elagolix, an oral GnRH antagonist. N Engl J Med. 2017;377(1):28-40. https://doi.org/10.1056/NEJMoa1700089.

19. Tundia N, Hass S, Fuldeore MJ, Wang L, Cavanaugh T, Boone $\mathrm{J}$, et al. Validation and U.S. population norms of health-related productivity questionnaire. Value Health. 2014;18(3):A24.

20. Buono JL, Carson RT, Flores NM. Health-related quality of life, work productivity, and indirect costs among patients with irritable bowel syndrome with diarrhea. Health Qual Life Outcomes. 2017;15(1):35. https://doi.org/10.1186/s12955-017-0611-2.

21. Stewart WF, Ricci JA, Chee E, Morganstein D, Lipton R. Lost productive time and cost due to common pain conditions in the US workforce. JAMA. 2003;290(18):2443-54. https://doi. org/10.1001/jama.290.18.2443.

22. Surrey ES, Soliman AM, Johnson SJ, Davis M, Castelli-Haley J, Snabes MC. Risk of developing comorbidities among women with endometriosis: a retrospective matched cohort study. J Womens Health (Larchmt). 2018;27(9):1114-23. https://doi.org/10.1089/ jwh.2017.6432.

23. Touboul C, Amate P, Ballester M, Bazot M, Fauconnier A, Darai E. Quality of life assessment using EuroQOL EQ-5D questionnaire in patients with deep infiltrating endometriosis: the relation with symptoms and locations. Int J Chronic Dis. 2013;2013:452134. https://doi.org/10.1155/2013/452134.

24. Surrey ES, Soliman AM, Agarwal SK, Snabes MC, Diamond MP. Impact of elagolix treatment on fatigue experienced by women with moderate to severe pain associated with endometriosis. Fertil Steril. 2019. https://doi.org/10.1016/j.fertnstert.2019.02.031.

25. Moradi M, Parker M, Sneddon A, Lopez V, Ellwood D. Impact of endometriosis on women's lives: a qualitative study. BMC Womens Health. 2014;14:123. https://doi. org/10.1186/1472-6874-14-123.

26. Whelan E. 'No one agrees except for those of us who have it': endometriosis patients as an epistemological community. Sociol Health Illn. 2007;29(7):957-82. https://doi.org/10.111 $1 / \mathrm{j} .1467-9566.2007 .01024 . x$. 